\title{
Inhalt / Sommaire
}

Zur Erinnerung an Heinrich Buess 1911-1984 3

Andreas-Holger Maehle, Johann Jakob Wepfers experimentelle Toxikologie $\quad 7$

Adolf Faller, Zur Diskussion um das Stensen-Experiment 19

Luigi Belloni, Il «tumore» endorbitario del Feldmaresciallo Radetzky guarito «dalla terapia omeopatica» $\quad 35$

Samuel S. Kottek, Physicians and healing personnel in the works of Flavius Josephus 47

Pieter Smit, The Swiss zoologist Rudolf Burckhardt (1866-1908), pioneer in biohistory 67

Beat Rüttimann, Ein militärmedizinisches Examen im Jahre 1794. 85

Luzius von Rechenberg und Huldrych M. Koelbing, Hufelands Opiumtherapie im zeitgenössischen Vergleich

Werner Sackmann, James Israel: Meine Reise zum Sultan (1915), Bemerkungen zum Tagebuch einer ärztlichen Mission

Charlotte Triebel-Schubert, Das naturphilosophische Konzept der Physiologie bei Johann Christian Friedrich Harless

Huldrych Koelbing, Hippokratische Medizin - (Cinquième Colloque International Hippocratique) - ein internationales Kolloquium in Berlin

Urs Boschung, Zur Edition des Briefwechsels Haller-Bonnet

Buchbesprechungen/Book reviews

Otto Baur, Otto Glandien, Zusammenhang. Festschrift für Prof. Dr. Dr. Marielene Putscher (Erwin H.Ackerknecht)

George Rosen, The Structure of American Medical Practice 1875-1941 (Erwin H. Ackerknecht)

J. M. Lopez Pinero, M. Seoane y la introduccion en España del sistema sanitario liberal (Erwin H. Ackerknecht)

Werner H.Stöcklin, Toktok. Am Rand der Steinzeit auf Neuguinea (Erwin H. Ackerknecht)

Mary A.B. Brazier, A History of Neurophysiology in the 17th and 18th Centuries (Antoinette Stettler)

Arthur E. Imhof (Hrsg.), Der Mensch und sein Körper von der Antike bis heute (Carl Haffter)

Leopoldina-Meeting, Biologische Grundlagen der Geschichtlichkeit des Menschen (Carl Haffter)

Herbert Will, Die Geburt der Psychosomatik. Georg Groddek, der Mensch und Wissenschaftler (Carl Haffter)

Danielle Gourevitch, Le Mal d'être Femme. La femme et la médecine dans la Rome antique (Carl Haffter) 
Helmut Vogt, Der Arzt am Krankenbett (Carl Haffter) 186

F. K. Mathis, Ist die schwarze Köchin da? Alte Kinderspiele (Carl Haffter) 186

Axel Hinrich Murken, Lehrbuch der Medizinischen Terminologie (Carl Haffter) 187

Roche Lexikon Medizin (Carl Haffter) 188

Walter Pagel, The Smiling Spleen. Paracelsianism in Storm and Stress (Willem F. Daems) 188

Die Heilmittel der Hildegard von Bingen, übersetzt v. M.-L. Portmann (Willem F. Daems) 189

François Ledermann, Bibliographie des ouvrages suisses de pharmacopée

(Willem F. Daems)

Perspektiven der Pharmaziegeschichte. Festschrift für Rudolf Schmitz (Willem F. Daems) 192

Franz-Josef Kuhlen, Zur Geschichte der Schmerz-, Schlaf- und Betäubungsmittel in Mittelalter und früher Neuzeit (Guido Sigron)

Arndt Fleischer, Patentgesetzgebung und die chemisch-pharmazeutische Industrie im deutschen Kaiserreich (Gottfried Schramm)

U. Grass, Zu Leben und Werk von Jakob Reinbold Spielmann (Gottfried Schramm) 196

A History of Pharmacy in Pictures (Gottfried Schramm)

Wolfgang Hömberg, Der norddeutsche Bronzemörser im Zeitalter von Gotik und

Renaissance (François Ledermann)

Nachruf: Karl Eduard Rothschuh 1908-1984 